\title{
Adaptive Selective State-Transition Decoding: A Combined Approach for Trellis Coded Modulation Scheme
}

\author{
Niranjan B. S. ${ }^{1}$, Vanaja Shivakumar ${ }^{2}$ \\ ${ }^{I}$ Department of Electronics and Communication Engineering, B.M.S. College of Engineering, Bangalore, India \\ ${ }^{2}$ Department of Electronics and Communication Engineering, DON BOSCO Institute of Engineering and \\ Technology, Bangalore, India
}

\begin{abstract}
We are presenting a new suboptimum decoding strategy, namely, Adaptive Selective StateTransition Decoding (ASSTD) for Trellis Coded Modulation schemes transmission over band-limited ISI channels. The ASSTD is a combined approach provides an improved error performance over Reduced State Sequence Estimation (RSSE) techniques which are in practical use. The ASSTD operates on two different concepts and has the flexibility to work with a controlled complexity. Simulation results are obtained for static band-limited ISI channels. The results show that the ASSTD can be extended to fading channels.
\end{abstract}

Keywords - Adaptive, Parameter, Selective, Selection, Transition

\section{INTRODUCTION}

With the advancement in technology, increasing demand for reliable high rate digital data transmission demands coded communication systems having large spectral efficiency. It is the pioneer work of Dr. G. Ungerboeck in this direction who invented Trellis Coded Modulation (TCM) schemes [1,2]. TCM is a bandwidth efficient coded modulation technique developed for high rate digital data transmission. It is a combined modulation technique which has the ability to improve the robustness of digital transmission over Additive White Gaussian Noise (AWGN) channel by 3 to $6 \mathrm{~dB}$ relative to uncoded system. TCM also provides an improved error performance in the presence of other channel impairments. Some of the applications of TCM are: ASDL, satellite communications, WiFi, WiMAX, CD writing and in flash memories.

\section{TRELLIS CODED MODULATION SCHEME}

The Fig. 1 depicts the TCM encoder. It comprises a convolutional encoder of rate $\tilde{m} / \tilde{m}+1$ where $m$ is the number of bits to be transmitted and $\tilde{m} \leq m$ is the number of bits fed to the convolutional encoder. Resulting $\tilde{m} / \tilde{m}+1$ coded bits and $m-\tilde{m}$ uncoded bits form the trellis code. A redundancy of one bit is introduced in the code generated. The encoded $m+1$ bits are mapped into a signal point of M-QAM constellation where $M=2^{m+1}$. The encoded bits divide the signal constellation into subsets each of size $2^{m-\tilde{m}}$ from which one of the signal point is selected by the $m-\tilde{m}$ uncoded bits for transmission, and the concept is termed as set-partitioning. Since the convolutional encoder is a finite state machine the trellis codes generated are represented by a trellis structure where the nodes represent the encoder states and the transitions between specific nodes is the encoder state changes, depending upon the current information bits and previously generated symbol. Each state transition is associated with possible symbols subset that can be transmitted. The Trellis encoder generates sequences of symbols which are inter-dependent. This property is being used by the receiver for errorless symbol detection.

The MLSE is the optimum decoding strategy for TCM schemes $[1,2,3]$ in the presence of AWGN. The MLSE implemented through Viterbi Algorithm traces the encoder trellis to perform ML sequence estimation based on the noisy received signal $r(n)$ given by:

$$
r(n)=a(n)+w(n)
$$

where $a(n)$ is the transmitted symbol, $w(n)$ is the Additive White Gaussian Noise sample and $r(n)$ is the noisy received symbol. The received signal sequence $\{\mathrm{r}(\mathrm{n})\}$ is decoded into one of the allowed sequences $\left\{a_{i}(n)\right\}$ based on the optimum decoding rule. Accordingly, $a_{i}(n)$ is the selected sequence if:

$$
\mathrm{p}\left(r(n) / a_{i}(n)\right)>\mathrm{p}\left(r(n) / a_{j}(n)\right) \text { for all } i \neq j
$$

For an AWGN channel this is equivalent to computing the squared ED between $\left\{a_{i}(n)\right\}$ and $\{r(n)\}$ selecting the signal sequence for which: 

$\left.\left\{r(n)-a_{i}(n)\right\}\right|^{2}<\left|r(n)-a_{j}(n)\right|^{2} \quad$ for all $i \neq j$

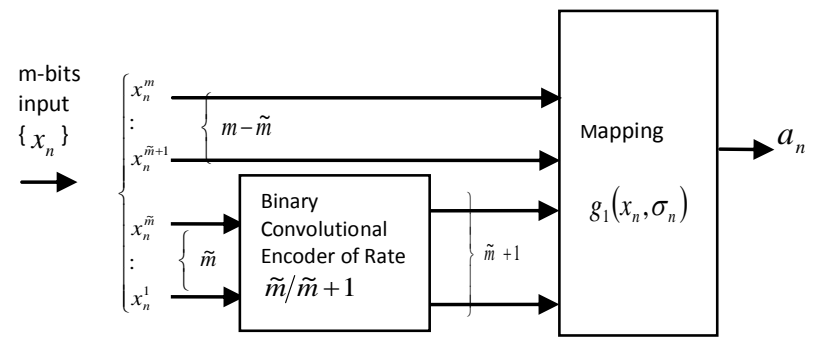

Fig. 1 General Structure of TCM

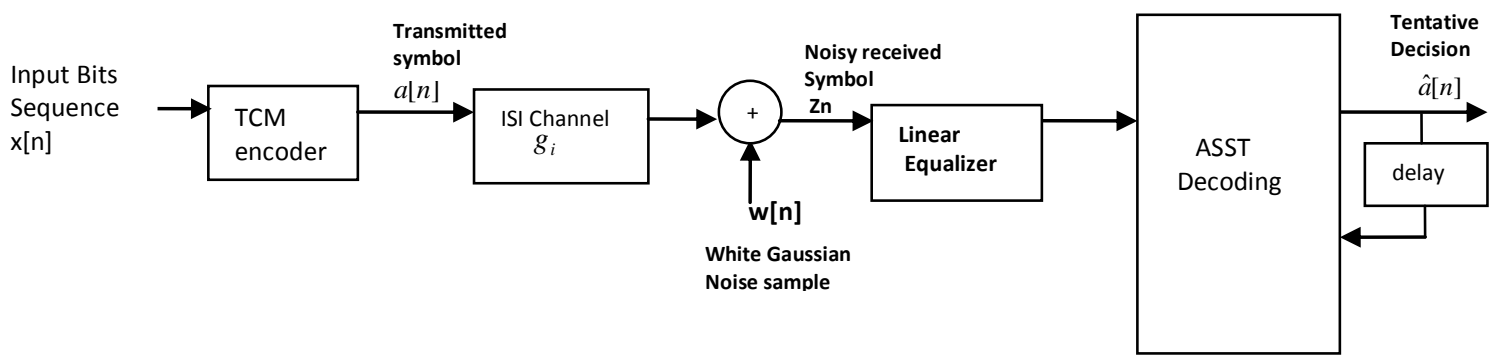

Fig. 2 Discrete-time model of data transmission system with ASSTD

The Viterbi decoder performs symbol decision after a delay of $\delta \geq 5 v$ where $v$ is the constraint length of the encoder. For band-limited ISI channels the maximum-likelihood sequence estimation has to process the ISI-Code trellis structure. The fact that the computational complexity of MLSE prohibits its implementation for bandlimited ISI channels initiated an era for the development of reduced complexity suboptimum decoding strategies. Among many suboptimum decoding techniques developed, Reduced State Sequence Estimation (RSSE) [4,5] is the one which finds many practical applications. In RSSE the ISI-Code trellis structure is reduced by merging the trellis states $[4,5]$. The metric computation in RSSE is given by:

$$
\tilde{M}_{n}=\min \left\{\tilde{M}_{n-1}+\left|z_{n}-\sum_{i=J+1}^{L} g_{i} \hat{a}_{n-1}-a_{n} g_{0}\right|^{2}\right\}
$$

where $\tilde{M}_{n}$ is the metric computation at the interval $\mathrm{n}, z_{n}$ is the noisy received symbol subjected to intersymbol interference and $\mathrm{L}$ is the channel memory length and $g_{i}$ are the channel impulse responses. The second term results in an error propagation in the feedback process due to the tentative decision $\hat{\boldsymbol{a}}_{n-1}$. The ASSTD minimizes this error propagation by incorporating adaptive noise minimization strategy.

\section{AdPTIVE SELECTIVE STATE-Transition DECODING (ASSTD)}

The ASSTD is a new suboptimum decoding strategy presented in this paper, for TCM schemes transmission over bandlimited ISI channels. Fig. 2 shows the block diagram of the communication The ASSTD combines two different concepts: firstly, it provides an improved error performance through fine tuning of adaptive coefficients $\lambda_{i}$ of the decoding algorithm, secondly, computational complexity is reduced by making the metric computations at various nodes of the trellis structure selective by incorporating selective statetransition [6]. The execution time is a function of the selectivity criteria being incorporated. Thus the ASSTD strategy provides the best possible error performance over the RSSE scheme within its error parameters constraints defined. The modified metric:

$$
\tilde{M}_{n}^{S}=\min \left\{\tilde{M}_{n-1}^{S}+\left|z_{n}-\sum_{i=J+1}^{L} g_{i} \hat{a}_{n-1}-\sum_{i=J+1}^{L} \lambda_{i} \hat{a}_{n-1}-a_{n} g_{0}\right|^{2}\right\}
$$

where $\tilde{M}_{n}^{S}$ is the metric computed with adaptive and selective state-transition approach. 


\section{CONCLUSION}

We have developed the new reduced complexity decoding strategy ASSTD for TCM schemes transmission over bandlimited ISI channel. Simulation results are obtained for 4-state 16-QAM TCM scheme transmission over two static ISI channels, with the impulse responses: $\mathrm{g} 0=.5, \mathrm{~g} 1=.5$ and $\mathrm{g} 0=.6$, g1=.4. Simulations results given in TABLE 1 and TABLE 2 show that the ASSTD provides improved error performance over RSSE. Hence the technique can be adapted for TCM schemes in application where Noise effect is severe, and in applications where TCM is a component code of a concatenated code.

It is also noted that the gain improvement is a function of the tuning process of the adaptive algorithm incorporated in ASSTD, symbol error improvement is shown in "Adaptive Minimization" column of the TABLE 1 and TABLE 2. The selective likelihood estimation is another factor of ASSTD which dominantly defines the computational complexity of the algorithm which in turn decides the execution time. The graph shown in Fig.3 depicts normalized execution time Vs SNR in dB. From the top, curve number 1 is the execution time of Adaptive minimization process in the absence of selective state-transition. The second curve from top is for the simplest case of RSSE that is PDFD. The third curve is for ASSTD strategy for selective state-transition coefficient $\mathrm{S}=0.2 \mathrm{~V}$ where $\mathrm{V}$ is noise variance, and the bottom curve depicts the normalized time of execution of ASSTD for S=V. The ASSTD has the flexibility to adapt to both the concepts in accordance with the noise parameters. Consequently the ASSTD can be used in TCM applications related to fading channels as well.

Table 1

Table showing the symbol error as a function of selective state transition parameter $\mathrm{S}$, for $\mathrm{g} 0=.6, \mathrm{~g} 1=.4$

\begin{tabular}{|c|c|c|c|c|c|c|}
\hline \multirow{2}{*}{$\begin{array}{l}\text { SNR } \\
\text { in } \\
\text { dB }\end{array}$} & \multicolumn{2}{|c|}{$\begin{array}{l}\text { No. of State discarded selective } \\
\text { state-transition approach as a } \\
\text { function of selective coefficient } S \\
\text { in terms of noise variance V }\end{array}$} & \multicolumn{2}{|c|}{$\begin{array}{c}\text { Symbol Error in } \\
\text { ASSTD }\end{array}$} & \multirow{2}{*}{$\begin{array}{c}\begin{array}{c}\text { Symbol Error in } \\
\text { PDFD }\end{array} \\
\text { Symbol Error }\end{array}$} & \multirow{2}{*}{$\begin{array}{c}\text { Symbol Error in } \\
\text { Adaptive } \\
\text { minimization }\end{array}$} \\
\hline & For $\mathrm{S}=\mathrm{V}$ & For $S=0.2 \mathrm{~V}$ & For $S=V$ & For $\mathrm{S}=0.2 \mathrm{~V}$ & & \\
\hline 18 & 2 & 0 & 79 & 74 & 94 & 80 \\
\hline 17 & 17 & 3 & 379 & 356 & 367 & 357 \\
\hline 16 & 38 & 4 & 1443 & 1407 & 1420 & 1410 \\
\hline 15 & 108 & 6 & 3833 & 3680 & 3821 & 3675 \\
\hline
\end{tabular}

Table 2

Table showing the symbol error as a function of selective state transition parameter $\mathrm{S}$, for $\mathrm{g} 0=.5, \mathrm{~g} 1=.5$

\begin{tabular}{|c|c|c|c|c|c|c|}
\hline \multirow{2}{*}{$\begin{array}{l}\text { SNR } \\
\text { in } \\
\text { dB }\end{array}$} & \multicolumn{2}{|c|}{$\begin{array}{l}\text { No. of State discarded selective } \\
\text { state-transition approach as a } \\
\text { function of selective coefficient } S \\
\text { in terms of noise variance } \mathrm{V}\end{array}$} & \multicolumn{2}{|c|}{$\begin{array}{l}\text { Symbol Error in } \\
\text { ASSTD }\end{array}$} & \multirow{2}{*}{$\begin{array}{c}\begin{array}{c}\text { Symbol Error in } \\
\text { PDFD }\end{array} \\
\text { Symbol Error }\end{array}$} & \multirow{2}{*}{$\begin{array}{c}\begin{array}{c}\text { Symbol Error in } \\
\text { Adaptive } \\
\text { minimization }\end{array} \\
\text { Symbol Error }\end{array}$} \\
\hline & For $\mathrm{S}=\mathrm{V}$ & For $S=0.2 \mathrm{~V}$ & For $\mathrm{S}=\mathrm{V}$ & For $S=0.2 \mathrm{~V}$ & & \\
\hline 19 & 0 & 0 & 108 & 102 & 102 & 96 \\
\hline 18 & 3 & 0 & 345 & 322 & 328 & 311 \\
\hline 17 & 14 & 2 & 1641 & 1632 & 1741 & 1621 \\
\hline 16 & 34 & 2 & 4621 & 4580 & 4754 & 4574 \\
\hline 15 & 96 & 7 & 10813 & 10710 & 11122 & 10698 \\
\hline
\end{tabular}




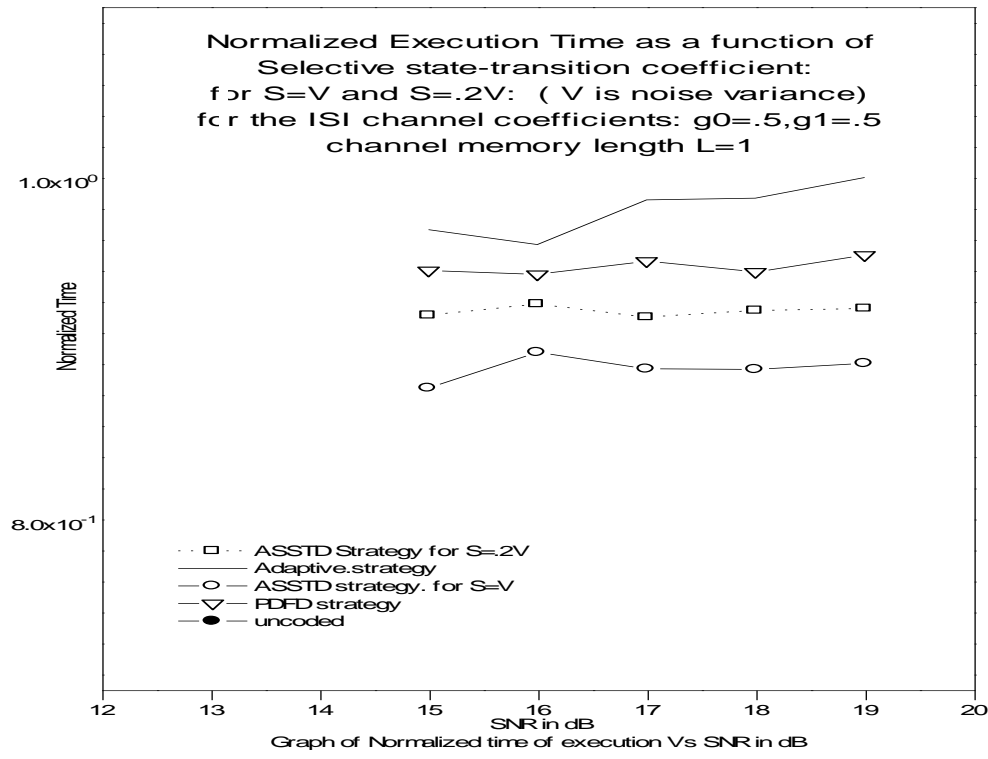

Fig.3 Normalized time of execution Vs SNR in dB for

PDFD, ASSTD and Adaptive minimization strategies, for channel memory L=1

\section{Acknowledgements}

The authors would like to thank the review committee members. We also thank Mr. Shivakumarappa. B. S. for all kind of co-operations extended in bringing out this paper.

\section{Journal Papers:}

\section{REFERENCES}

[1] Gottfried Ungerboeck, "Trellis-Coded Modulation with Redundant Signal Sets part1: Introduction," IEEE communications magazine, Feb. 1987-vol.25, No. 2

[2] Gottfried Ungerboeck, "Trellis-Coded Modulation with Redundant Signal Sets part1I: State of the art," IEEE communications magazine, Feb. 1987-vol.25, No. 2

[3]. Forney G.D., Jr.,"Maximum-likelihood Sequence Estimation of Digital Sequences in the Presence of Intersymbol Interference," IEEE trans. Inform. Theory, Vol. IT-18, pp. 363-378, May 1972.

[4]. Pierre R. Chevillat and Evangelos Elefthrious, "Decoding of Trellis-Encoded Signals in the presence of Intersymbol Interference and Noise," IEEE Transaction on communications, vol 37. No.7, July 1989

[5]. M. Vedat Eyboglu and Shahid U. H. Qureshi, "Reduced -State Sequence Estimation for Coded Modulation on Inter -symbol Interference Channels," IEEE Journal on Selected Area in communications, Vol. 7, No. 6, Aug 1989.

[6]. Niranjan. B. S. ,Vanaja Shivakumar, International Journal of Engineering Research \& Technology (IJERT) Vol. 1Issue 5, July-2012 ISSN: 2278-0181

\section{Books:}

[7]. Proakis J. G., Digital Communications New York: McGraw- Hill,1989, $3^{\text {nd }}$ ed. 